\title{
Chronique bibliographique. A propos de Crises extrêmes. Face aux massacres, aux urgences civiles et aux génocides
}

Francesco Ragazzi

\section{(2) OpenEdition \\ Journals}

Édition électronique

URL : http://journals.openedition.org/conflits/2211

DOI : $10.4000 /$ conflits.2211

ISSN : 1777-5345

Éditeur :

CCLS - Centre d'études sur les conflits lilberté et sécurité, L'Harmattan

Édition imprimée

Date de publication : 1 mai 2007

Pagination : 168-172

ISBN : 978-2-296-03084-8

ISSN : 1157-996X

Référence électronique

Francesco Ragazzi, « Chronique bibliographique. A propos de Crises extrêmes. Face aux massacres, aux urgences civiles et aux génocides ", Cultures \& Conflits [En ligne], 65 | printemps 2007, mis en ligne le 04 janvier 2010, consulté le 30 mars 2021. URL : http://journals.openedition.org/conflits/2211 ; DOI : https://doi.org/10.4000/conflits.2211 


\title{
Chronique bibliographique
}

\author{
Le Pape M., Siméant J., Vidal C., Crises extrêmes. Face aux massacres, \\ aux urgences civiles et aux génocides, Paris, La Découverte, 2006.
}

\section{Francesco RAGAZZI}

Francesco Ragazzi est doctorant en sociologie des Relations internationales à l'IEP de Paris et à Northwestern University. Sa recherche porte sur les politiques de " diaspora "t le conflit en ex-Yougoslavie.

T 'ouvrage collectif Crises extrêmes s'inscrit dans le cadre du Groupe de recherLche éponyme co-dirigé depuis 2003 par Marc Le Pape, Johanna Siméant et Claudine Vidal. Il est le fruit d'un travail interdisciplinaire réunissant sociologues, juristes, anthropologues, politistes et philosophes et dont le but est d' "observer non pas la logique propre des crises mais les modalités selon lesquelles elles sont constituées en objets de connaissance, de controverses, de mobilisations et d'actions $^{1}{ }^{»}$. Les génocides, les massacres, les meurtres de masse sont en effet appréhendés par une série de discours discordants, de natures différentes, qui sont inévitablement liés au contexte social, professionnel et politique dans lequel ils sont formulés. Mais ces discours ne sont pas seulement des « mots », ce sont des faits, des « choses » qui déterminent le sort d'un réfugié, le déclenchement d'une action humanitaire, ou la préparation d'une intervention armée. Cet ouvrage retrace les cadres sociaux dans lesquels se formulent ces énoncés tout en étant attentif, dans des contextes dominés par l'incertitude, à établir non pas « une vérité » scientifique sur les situations de crises extrêmes, mais bien plutôt les pratiques de véridiction des différents acteurs sociaux engagés.

L’ouvrage est divisé en deux parties. La première, «Qualifier, mettre en récit, représenter » se concentre sur l'élaboration de différents discours et de leur capacité à rendre compte, à construire un récit portant sur des situations de crises extrêmes. Cela vaut autant pour le discours journalistique et médiatique

1. Le Pape M., Siméant J., Vidal C., Crises extrêmes. Face aux massacres, aux urgences civiles et aux génocides, Paris, La Découverte, 2006, p. 11. Toutes les citations de cet article sont tirées de cet ouvrage. 
(Claudine Vidal, Johanna Siméant, Pascal Dauvin, Sophie Pontzeele, Alice Krieg-Planque) que celui des gouvernements et des ONG (Marc Le Pape, David Garibay et Juan-Carlos Guerrero-Bernal, Michel Agier) et enfin du discours universitaire et juridique (André Guichaoua). La seconde partie, intitulée « Formes et raisons de l'intervention ", s'interroge sur la capacité de tels discours à déclencher des pratiques d'intervention politique dans ces contextes de crises et ce, qu'il s'agisse des catégorisations et pratiques bureaucratiques (Arnaud Royer, Yves Buchet de Neuilly), des discours tenus par les ONG (Rony Brauman, Didier Fassin et Estelle D’Halluin, Karine Vanthuyne, Eric Poinsot, Isabelle Delpla) ou encore le discours universitaire (Sandrine Lefranc).

Il est difficile, face à la diversité des contributions et à l'étendue des zones géographiques traitées (Biafra, Bosnie-Herzégovine, Burundi, Cambodge, Colombie, Guatemala, Kosovo, Liberia, Palestine, RDC, Rwanda) de rendre compte de la complexité des argumentations de chacun des auteurs. On privilégiera donc une analyse thématique plutôt que la présentation exhaustive des argumentaires de chaque auteur.

\section{Le témoignage}

La figure du témoin est, selon les trois co-directeurs de l'ouvrage, «inhérente aux perceptions publiques des crises extrêmes ${ }^{2}$ ». C'est en effet généralement à travers le témoignage, ou l'ensemble des témoignages, qu'apparait la réalité d'une situation « extrême ». L'ensemble des contributions de l'ouvrage portant sur la question du témoignage souligne le caractère fondamentalement dialogique de la pratique du témoignage. Un témoignage est, en effet, toujours destiné à être entendu par un individu, une institution, un tribunal, un journaliste ou une ONG. C'est à la fois une pratique de construction d'une vérité historique et une mise en récit qui permet à la victime, au bourreau, ou au témoin de retrouver sa dignité sociale, d'être réinstallé dans un autre pays, ou encore de s'acquitter d'une accusation. Que ce soit dans le cadre des commissions de "Vérité, justice et réconciliation » ou dans le cas de la détermination du statut de réfugié, la forme donnée au passé conditionne les dénouements futurs ${ }^{3}$. En ce sens, une analyse du témoignage décentre le chercheur de «[...] la question de la vérité vers la question du (et des) pouvoir(s) - des pouvoirs de la parole en particulier ${ }^{4}$ ».

La parole n'est cependant pas la forme exclusive par laquelle le témoignage s'insère dans les pratiques d'élaboration des "vérités ». Comme le montre le cas des massacres en Bosnie-Herzégovine, les corps mêmes des victimes "témoignent» des massacres. Bien que ne disposant que peu d'information sur leurs modalités, les disparitions sont une certitude et, de fait, constituent 
un témoignage en elles-mêmes. Les postures physiques des corps lors de leur exhumation (mains liées, traces d'impact de balles derrière la nuque) « témoignent » des exactions commises 5 . Autre forme non discursive de témoignage, la stratégie du silence, par exemple, ces témoins, dont les récits ne permettent pas de les classer dans des catégories victimes/bourreaux par trop dichotomiques telles ces «jeunes filles violées devenues épouses de leurs agresseurs soldats ou miliciens, celle des villageois prisonniers devenus combattants [...]. Pourquoi parler puisqu'il y a de bonnes raisons de se taire ? 6 ».

Mais le témoignage permet cependant de rompre un silence imposé et de restituer l'expérience des victimes dans un contexte collectif où leur passé prend un sens. Un sens aux yeux mêmes des sujets, comme un effort de mémoire qui s'insère dans une mémoire collective, mais qui prend également un sens politique et juridique. Et c'est précisément à travers ce travail de traduction de l'expérience individuelle en un langage effectif et «efficace» dans le champ juridique, humanitaire ou politique et une nécessaire socialisation avec le "langage autorisé ", ou du moins familier à ces institutions, que « dire » permet ultimement de « faire».

\section{Les porte-paroles}

De nombreuses organisations se donnent pour mission de traduire la parole des témoins en un langage "efficace». En fonction des contextes politiques et des stratégies des ONG, les discours sont traduits en revendications et termes juridiques précis et facilement assimilables par un tribunal (comme le TPIY), ou encore par les acteurs humanitaires, tel le HCR, pour faciliter le déclenchement d'opérations de soutien. Pour le Centre d'action légale pour la défense des droits humains (CALDH) au Guatemala par exemple,

«La première fois qu'on a rassemblé des témoins des massacres, ils avaient le regard rivé au sol, il ne se regardaient pas, il avaient peur de parler, et c'est peu à peu, en brisant la glace, qu'ils ont commencé à raconter leur histoire. C'est super-émotionnel [...]. Maintenant, $[\ldots .$.$] les gens posent des questions telles que : "Si notre poursuite est$ rejetée par le ministère public, pouvons-nous la porter devant la Commission inter-américaine des droits de l'Homme ?" (Susanna, CADLH) ${ }^{7}$ ».

Le discours du témoin est donc une pratique sociale en dialogue avec des institutions pour qui le récit légitime le déclenchement d'une action. 
Mais il peut arriver que la parole des porte-parole supplante celle qu'elle est censée représenter. En effet, la position de «porte-paroles » et de dénonciation qui marque la rupture des ONG telles que Médecins sans frontières avec les pratiques de silence du Comité international de la Croix-Rouge utilise précisément la force rhétorique de "vérité » de sa position de témoin-organisation dans le jeu politique, en masquant cependant ses intérêts propres. Ici, la « vérité » du témoin est remplacée par la « vérité » du « secouriste-narrateur 8 », instrument de qualification des crises ${ }^{9}$ dont l'existence politique et financière dépend précisément de ces dernières. Les cas du Biafra et du Cambodge pour MSF, ou encore du poids des luttes internes d'Amnesty international dans la formulation du soutien aux interventions armées 10 montrent de nouveau l'importance de décaler le regard vers les logiques de pouvoir qui donnent naissance aux discours des ONG, et de replacer leur action dans le champ plus large des luttes et interactions qui caractérisent les organisations humanitaires. L'ouvrage attire en ce sens notre attention sur le fait que, contrairement à une idée reçue sur la « réactivité » des ONG face aux situations de crise extrêmes, c'est précisément les contraintes d'un champ de force structuré par les bailleurs de fonds, les gouvernements, les autres ONG et la base militante qui conditionne les «missions». C'est ainsi qu'au discours des droits de l'Homme et des principes humanitaires s'ajoute le discours psychologique du «traumatisme », qui, outre la dépolitisation des stratégies d'intervention, permet à certaines ONG comme MSF de s'implanter dans des pays ou l'aide médicale de première necessité (chirurgie, salles opératoires, médicaments, etc.) n'est pas requise. $\mathrm{La}$ « santé mentale » devient donc, pour le cas de la Palestine par exemple, un prétexte "pour y être ${ }^{11}$ ».

\section{Les médias}

Si les pratiques de dénonciation des ONG n'ont de sens qu'à travers leur relais par le discours journalistique, pour ce dernier, les témoignages directs et les témoignages des ONG ne représentent qu'une des sources parmi d'autres pour l'élaboration d'une « vérité » sur les enjeux d'une crise extrême. Mais ici encore, les contributions insistent sur les contraintes liées à la pratique de journalisme et de son format particulier dans l'élaboration de la «vérité ». Qu'il s'agisse d'une distinction entre «bons » et «mauvais » témoins, de l'interaction entre un reporter et sa rédaction, du repérage des «fixeurs », de la concurrence entre reportage et dépêche d'agence, l'écriture journalistique est un compromis entre exigences de vérité, conditions matérielles de vérification des témoignages et lignes éditoriales des rédactions ${ }^{12}$. C'est donc dans ce contexte complexe de production et de normes professionnelles, (la glorifica-

8. Voir p. 190.

9. Voir p. 150.

10. Voir p. 269.

11. Voir p. 210.

12 . Voir pp. 57-71. 
tion du «terrain », le «bon papier », «l'objectivité ») qu'il faut interpréter la production du discours journalistique. Ainsi, la nécessité de traduire en termes familiers des situations lointaines pousse les rédactions aux analogies ${ }^{13}$. C'est alors que le terme de "génocide ", mobilisant les répertoires d'énonciation de l'horreur nazie, vient décrire les situations burundaise et rwandaise ${ }^{14}$. De même, le souci d'impartialité, augmenté de la fascination pour les mots du terrain, aboutit à l'élaboration de discours exotisant («le génocide à la machette 15 ", la «purification ethnique 16 ", qui permettent de «dénoncer sans désigner », en brouillant les pistes des responsabilités).

\section{Institutions étatiques et organisations internationales}

Le discours médiatique façonné par ses conditions de production se trouve cependant pris dans un champ plus large, celui du pouvoir étatique. Contrairement aux idées reçues sur l'effet $\mathrm{CNN}$,

« de la même façon que les sociétés ne se posent jamais que les questions auxquelles elles peuvent répondre, les médias n’appellent jamais qu'aux interventions "humanitaires" dont ils anticipent que le politique a quelque chance de ne pas se désintéresser 17 ».

Le rôle des Etats et des organisations internationales est donc crucial. Il est déterminant dans sa capacité à produire une "vérité » sur la crise dans des catégories et formulations bureaucratiques qui affecteront directement le destin de milliers d'individus. Qu'il s'agisse du cas du Rwanda avec la distinction entre «déplacés Tutsi », «dispersés Hutus » et « regroupés », ces taxinomies incarnent des «biens symboliques sur lesquels les acteurs agrippent leurs stratégies 18 ", ou encore dans le cas des enquêtes parlementaires françaises et belges dans l'amoindrissement du rôle des gouvernements dans le génocide ${ }^{19}$. Enfin, comme pour les ONG, l'étude du cas de l'Union européenne et de sa stratégie de gestion de crise montre comment c'est plus à travers une logique de « coups » stratégiques, que de «capacité de réaction » qu'il faut comprendre l'activation de politiques particulières. Rarement en effet «l'objectivité des massacres 20 » coïncide avec les enjeux de luttes bureaucratiques qui façonnent la Politique européenne de sécurité et de défense (PESD).

\footnotetext{
13. Voir pp. 57-71.

14 . Voir p. 101.

15 . Voir p. 83.

16. Voir p. 21.

17. Voir p. 100.

18 . Voir p. 56.

19. Voir p. 172.

20. Voir p. 103.
} 


\section{Les chercheurs}

L'intérêt de cet ouvrage collectif tient enfin à l'intégration du regard réflexif du chercheur. Dans l'ambiguïté de sa position, par exemple entre sociologue et « témoin-expert » pour le Tribunal pénal international pour le Rwanda, le sociologue fait lui-même l'expérience de la multiplicité des « codages » possibles du témoignage, mettant ainsi en lumière l'importance cruciale de la question du « cadre » du discours, du choix du vocabulaire et de la grammaire du témoignage, entre «vérité procédurière » et «vérité du réel » 21. Mais dans une structuration où les champs universitaires et des praticiens sont relativement poreux, comme c'est le cas aux Etats-Unis, le discours universitaire acquiert une position d'autorité et ajoute une dimension supplémentaire à la dialectique savoirs-pouvoirs. C'est le cas des théories du «peace-building scientifique ", fondées sur la psychologie sociale et les théories du choix rationnel. La conception positiviste de la transmission de la science à son "application » permet en effet l'élaboration et le financement de projet de «peacebuiling " à travers le «confidence-building » et la «two-track diplomacy », justifiant des pratiques décontextualisées de réconciliation, forcément destinées à des résultats improbables ${ }^{22}$.

Les passages les plus faibles du livre se trouvent sans doute dans certaines contributions de la première partie, trop centrées sur une analyse interne du discours médiatique et ne prenant que peu ou pas du tout en considération les conditions matérielles de leur énonciation ou leurs usages politiques. Cela mis à part, il est difficile de trouver des brèches dans un ouvrage dont l'axe problématique central prend dans son fondement une posture radicalement critique vis-à-vis des différentes sources de production de «vérité » sur des événements souvent dominés par l'incertitude. Dans la plupart des contributions, la « parole » est restituée à la fois dans son contexte social d'apparition et évaluée dans sa capacité à se constituer en tant que levier d'activation des relations de pouvoir : de pouvoir individuel, journalistique, entrepreunarial (humanitaire) ou pouvoir étatique dans l'objectivation de (la situation de) populations ou d'une "vérité officielle ». Enfin, la dimension critique et réflexive de l'ouvrage permet une prise de distance des discours universitaires orientés vers le «problem-solving ", tributaire des positions et présupposés des praticiens, qui viennent souvent justifier des stratégies de multi-positionnement professionnel (Université, ONG, organisation internationale, cabinet de conseil). L'interrogation sur les conditions mêmes de "mises en récit » oblige à s'interroger sur les catégories même d'appréhension de ces processus politiques. 